\title{
Opacity simulation technique for confocal microscope image processing
}

\section{Miroslav Druckmüller}

Department of Mathematics, Faculty of Engineering, Brno University of Technology, Czech Republic

\begin{abstract}
Summary
3D-visualization of small transparent objects like cells by means of classical methods often gives 3D sensation insufficient for the understanding of objects topology. Method introducing artificial opacity are described in this article.
\end{abstract}

Keywords: 3D model - confocal microscope - opacity - transparent object

\section{INTRODUCTION}

Visualization of small objects like cells is a specific problem because the visible light $(400-700 \mathrm{~nm})$ has a relatively long wavelength in comparison to the object size. This fact causes several phenomena which make the understanding of the object shape rather uneasy (Martišek 2002c). Cells observed by a conventional or confocal microscope are often highly transparent and because of the lighting geometry the structures inside the cell do not create visible shadows. There are no visible reflections on surfaces which make it possible to understand surface curvature. Finally the contrast of important structures is often very low and the depth of the focussing small. All these problems together ensure that the $3 \mathrm{D}$ sensation of microscope images, even when made by a confocal microscope, is often very poor. It is for this reason that methods for $3 \mathrm{D}$ reconstruction of confocal microscope images are nowadays widely studied (Martišek and Mikulášek 2001, Martišek 2002a, 2002b, 2002e).

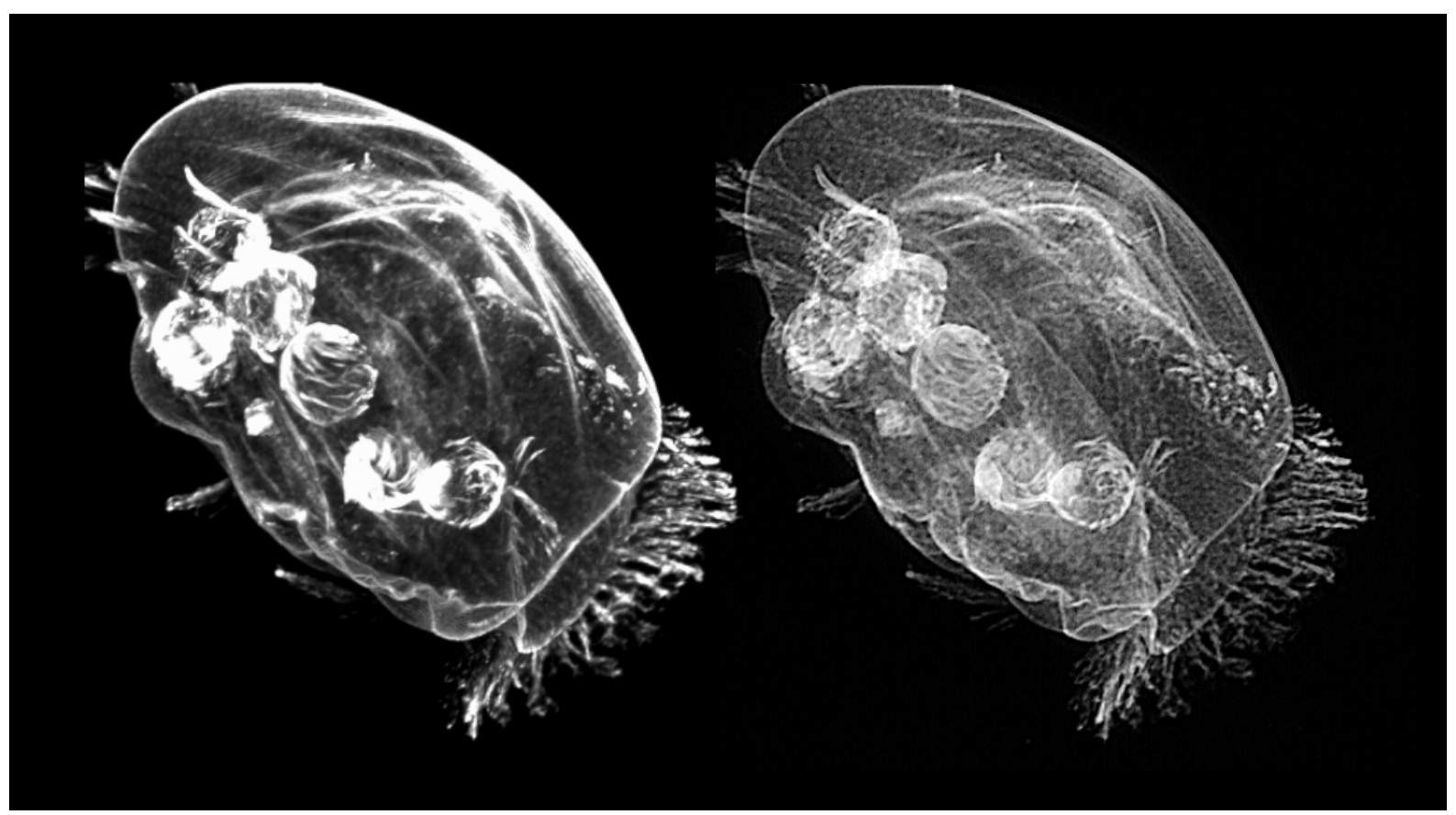

Fig. 1. Classical 2D composite image. The left cell image is created by means of a standard composition algorithm disjunction of all confocal cuts. The right cell image is created by means of an advanced ACC adaptive contrast control algorithm (Druckmüller 2001). As the cell is nearly perfectly transparent the observer can hardly guess the 3D topology of the cell according either to the left image or the right one. 


\section{Druckmüller}

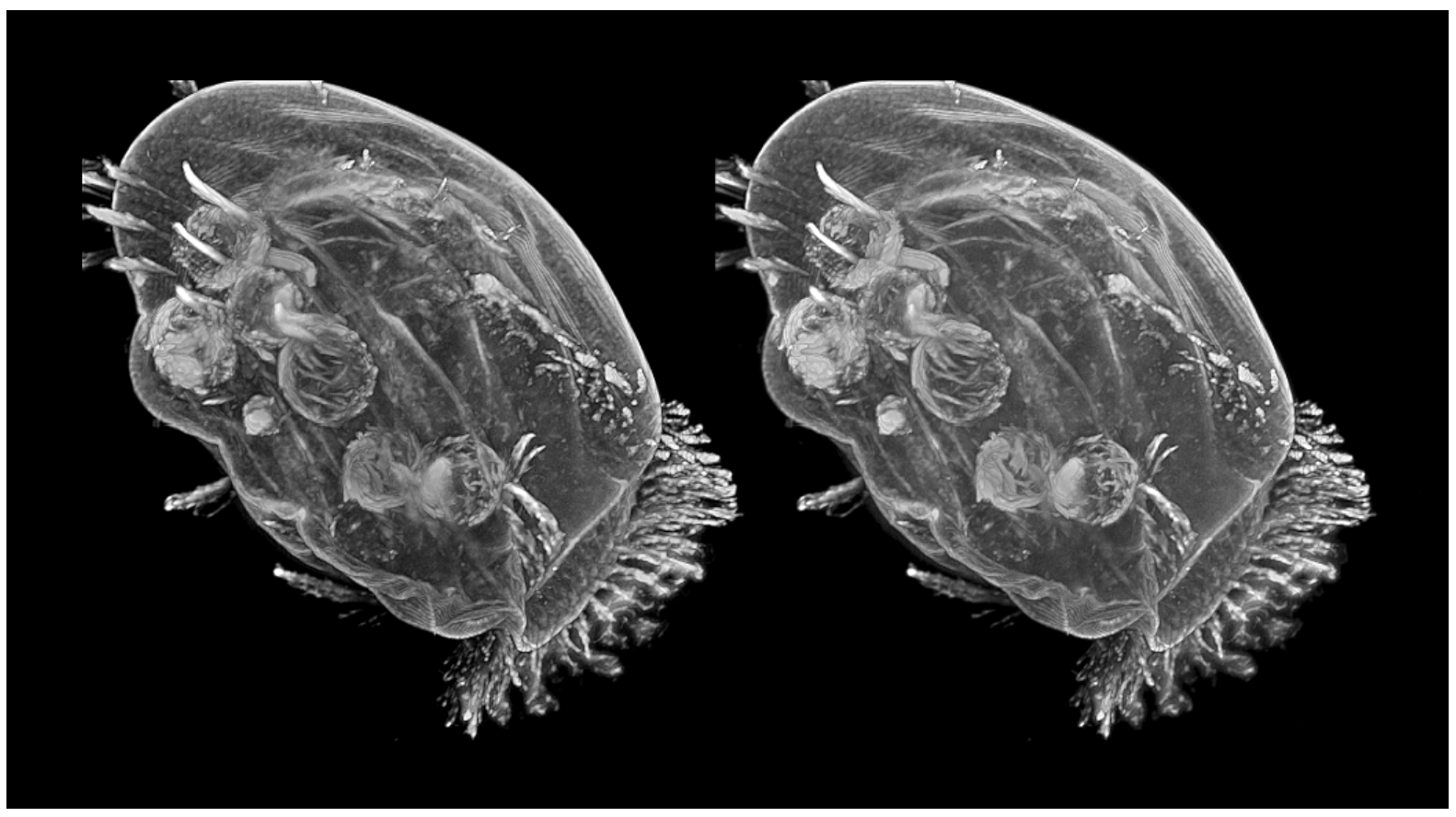

Fig. 2. 3D reconstruction of the cell made by the opacity simulation technique. The opacity of the cell was increased from nearly $0 \%$ in reality to $5 \%$ on the right image and to $20 \%$ on the left one. Even if the angle of view is perpendicular to the focussing plane, the image geometry is the same as in fig. 1 . The $3 \mathrm{D}$ sensation has increased and the contrast loss of structures inside the cell is negligible.

\section{D MODEL VISUALIZATION METHODS}

These methods are based on the segmentation of confocal microscope images and the creation of a 3D model (Martišek 2001, Martišek 2002d). All cell structures must be correctly identified and separated by means of a segmentation method. This makes it possible to create a 3D voxel model which can be visualized by means of wide choice of methods (Martišek 2002e). This method is nowadays the one most used. The high speed of 3D model visualization is the great advantage of this method. This speed is reached by computer hardware as nowadays graphic interfaces have special graphic instruction sets which accelerate the 3D visualization. Rotation of the object and changing of lighting conditions can be carried out in real time. The observer can see the object from an arbitrary direction and can obtain a very good 3D image of the object. The main problem with this method is the segmentation of confocal microscope images. Simple segmentation methods like thresholding often fail and rather complicated adaptive methods have to be used. Even these advanced segmentation methods are not able to handle the segmentation problem in all its complexity. It is often necessary to resort to the so called "manual" segmentation method i.e. the user has to draw object boundaries into the image subjectively. It decreases the scientific reliability of results.

3D matrix image processing - opacity simulating algorithm - is more exact than the previous method. However, it makes extremely high demands on computer memory and speed. It handles the set of confocal microscope images focused on various planes, so-called cuts, as 3D matrix. Pixel values of particular images can be interpreted not only as light intensity but various properties can be associated with pixel values like brightness, hue, density, opacity, reflexivity, light diffusion, etc.

These properties are dependent on pixel values and by setting the various dependency formulas we can simulate various 3D object attributes. This makes it possible to create 3D images of smalls objects more similar to those ones we are used to observing with the naked eye and therefore more comprehesible for human vision.

An especially successful algorithm is the so called opacity simulating algorithm. 


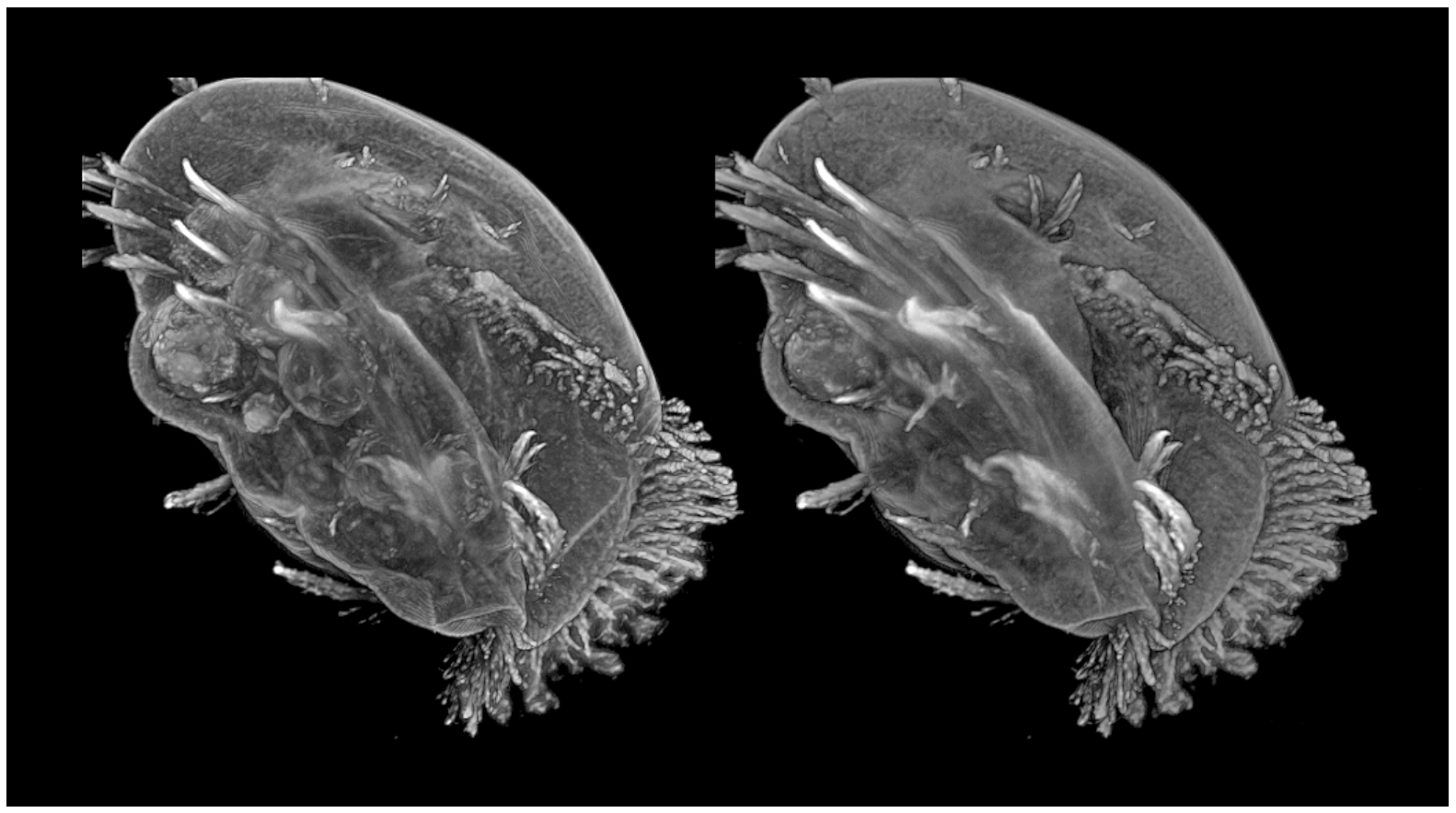

Fig. 3. Decreasing the cell opacity to $40 \%$ (fig. 3, left image) makes the 3D sensation even better in comparison to Fig. 2 but there is a visible loss of information in cell interior. The $60 \%$ value (this figure, right image) of simulated cell opacity represents probably the limit value for obtaining useful results as structures inside the cell are practically invisible.

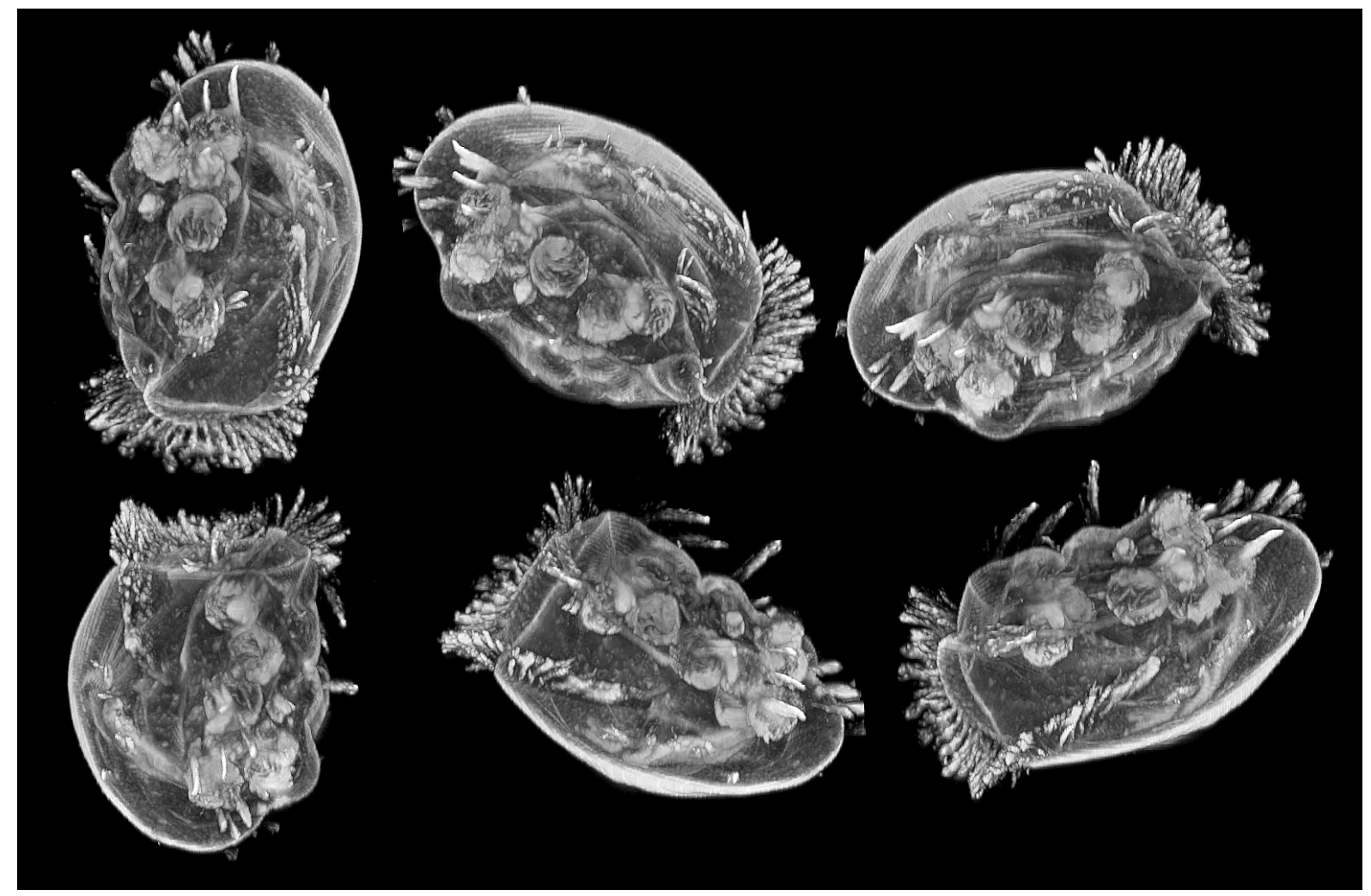

Fig. 4. Cell from arbitrary direction. It is obvious that none of the presented images are able to show the cell 3D structure in its full complexity. However, the opportunity to observe the cell from an arbitrary direction (this figure) withan arbitrary defined opacity makes it powerful tool for the understanding of 3D cell structure. Computer animation using the simulated opacity technique especially gives an excellent $3 \mathrm{D}$ sensation. 


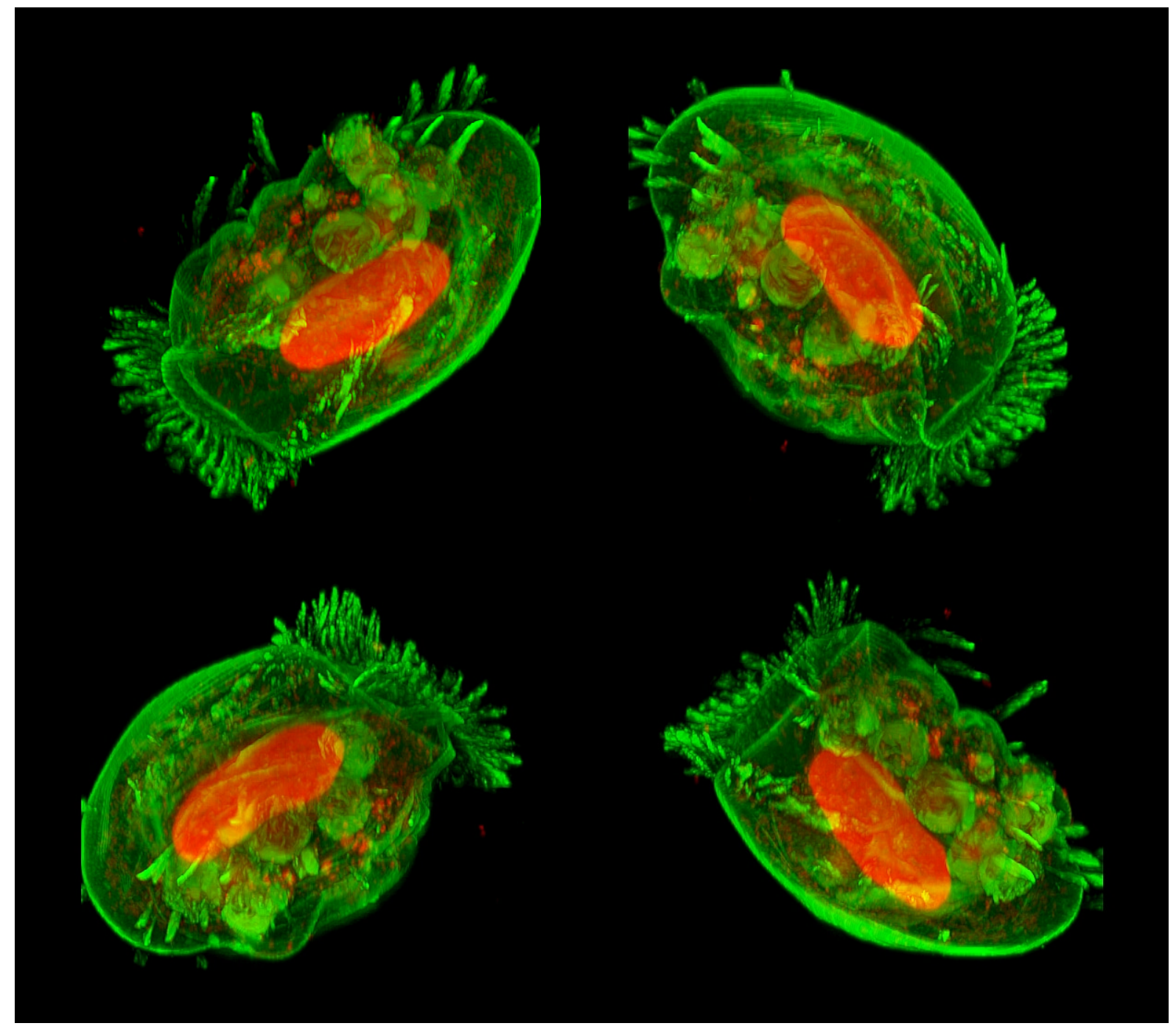

Fig. 5. Opacity simulation technique on the color image. The opacity simulation technique can be applied to color images also. The processing in this case consists of three steps. In the first step the color image is represented in LHS (lightness, hue, saturation) color space. The opacity simulation technique is applied on L component in the second step. Finally the image is converted back to the standard RGB color space. This procedure was used in the creation of a color image showing the rotation of a protozoan cell.

It gives good results by processing fluorescent confocal microscope images of cells. It is based on the idea that pixel value $\mathrm{x}$ determines local opacity $\mathrm{q}$ by formula $\mathrm{q}=\mathrm{f}(\mathrm{x})$, where $\mathrm{f}$ is a user defined function. It enables the changing of the cell opacity for nearly zero value (the cell is transparent) to $100 \%$ opacity (the cell is opaque and its interior is not visible). The following example illustrates the possibilities of $3 \mathrm{D}$ reconstruction of protozoan images made by a fluorescent confocal microscope with simulated cell opacity. Images were processed with an SOFO image analyzer ACC 5.0. The author of the primary data is Prof. R. Janisch (Departement of Biology, Faculty of Medicine, Masaryk University, Brno, Czech Republic).

\section{ACKNOWLEDGEMENT}

This study was supported by research design CEZ: J22/98:261100009 "Non-traditional methods for investigating complex and vague systems".

Received $27^{\text {th }}$ January 2003.

Published online $5^{\text {th }}$ March 2003. 


\section{REFERENCES}

Druckmüller D.: Adaptive Image Processing in Biology, In J. Berger (ed): Cells III, Kopp Publ. České Budějovice 2001, p. 41-45.

Martišek D and K. Mikulášek: Inverse mapping and its use for 3-D data visualisation, Energy and Information in Nonlinear Systems, Czech Society of Applied Electromagnetics, Brno 2001, p. 60 63.

Martišek D.: 3-D filters and their use for computer modelling, Aplimat, Bratislava 2002a, p. 291296.

Martišek D.: Matematické principy grafických systémů, Littera, Brno 2002b, pp. 297.
Martišek D.: The 2-D processing of images provided by confocal microscopes, Mendel Brno 2002c, p. 363-371.

Martišek D.:Methods of detection of equipotential surfaces in cell structures, In J. Berger (ed): Cells IV, Kopp Publ. České Budějovice, 2002d, p. 47-54.

Martišek D.:The 2-D and 3-D processing of images provided by conventional microscopes, Scanning 24: 284-297, 2002e.

Preliminary report was published in J. Berger (ed.): Cells IV, Kopp Publ. České Budějovice 2002, p. $41-45$

$\triangle$ Address:

Miloslav Druckmüller, Department of Mathematics, Faculty of Engineering, Brno University of Technology, Technická 2, 61669 Brno, Czech Republic; druck@mat.fme.vutbr.cz 